\begin{tabular}{l|l} 
REVISTA & $\begin{array}{l}\text { Revista Educación } \\
\text { ISSN: 0379-7082 } \\
\text { ISSN: 2215-2644 } \\
\text { revedu@ gmail.com } \\
\text { Universidad de Costa Rica } \\
\text { Costa Rica }\end{array}$
\end{tabular}

\title{
Homogeneizar la práctica de la modelación: un reto del sistema educativo colombiano
}

\author{
Olarte García, Julián Alejandro \\ Homogeneizar la práctica de la modelación: un reto del sistema educativo colombiano \\ Revista Educación, vol. 44, núm. 1, 2020 \\ Universidad de Costa Rica, Costa Rica \\ Disponible en: http://www.redalyc.org/articulo.oa?id=44060092024
}

Esta obra está bajo una Licencia Creative Commons Atribución-NoComercial-SinDerivar 3.0 Internacional. 


\title{
Homogeneizar la práctica de la modelación: un reto del sistema educativo colombiano
}

\author{
Standardizing the Use of Mathematical Models: A Challenge Faced by the Colombian Educational System \\ Julián Alejandro Olarte Garcia $\quad$ Redalyc: http://www.redalyc.org/articulo.oa?id=44060092024 \\ Universidad del Quindio, Armenia, Colombia, Colombia \\ jaolarteg@uqvirtual.edu.co \\ iD http://orcid.org/0000-0002-8972-582X
}

Recepción: 08 Abril 2019

Aprobación: 12 Noviembre 2019

\section{RESUMEN:}

El objetivo en esta investigación es motivar una reflexión crítica frente a la práctica de la modelación matemática en el país a partir de una experiencia pedagógica. Las personas participantes eran estudiantes y la docente en el nivel quinto de educación básica de un colegio colombiano. Para el estudio se adoptó un enfoque cualitativo y la información que se recolectó mediante entrevistas, observaciones y documentos escritos por las estudiantes, fue analizada por el practicante docente para evidenciar fortalezas y dificultades. Los resultados dan evidencia de que la modelación matemática usada integralmente, despierta en el estudiantado un mayor interés, gusto y mejor desempeño en el área de matemáticas. Se concluye que la calidad de los procesos de enseñanza y aprendizaje exige que docentes y directivos docentes promuevan espacios de investigación, análisis e indagación a través de material concreto y situaciones contextualizadas. Para ello se recomienda la aplicación de herramientas tecnológicas en el ámbito escolar utilizadas como medio facilitador de las actividades de modelación matemática, mayor capacitación, disponibilidad de recursos y apoyo institucional para la adecuada planificación del personal docente.

Palabras Clave: Estudiantado, Profesorado, Modelación matemática, Escuela primaria, Colombia.

\section{Abstract:}

This study aims to provoke critical thought regarding the use of mathematical models in the classroom based on a pedagogical experiment involving a group of fifth grade students and teachers at a school in Colombia. The study had a qualitative focus with data collected through interviews, observation and documents written by the students themselves, which was then analyzed by the teacher to identify strengths and weaknesses. According to the results, frequent use of mathematical modelling is stimulating for the students. As a result, students become more engaged, acquire a more favorable outlook towards mathematics and earn better grades in the subject. Among the conclusions, greater use of technology in the classroom is recommended to facilitate mathematical modeling. For quality teaching and learning, both teachers and staff administrators must promote opportunities for research, analysis and inquiry, using tangible examples and contextualized situations. The use of Information and Communications Technology (ICT) in the classroom is recommended to facilitate mathematical modeling activities with greater teacher training opportunities, availability of resources and institutional support for adequate planning.

KEYWORDs: Students, Teachers, Mathematical Modeling, Elementary School, Colombia.

\section{INTRODUCCIÓN}

Quizá una de las mayores preocupaciones de la educación matemática es lograr que el alumnado encuentre sentido a los contenidos y lograr articular los conocimientos matemáticos con los contextos cotidianos que emanan de otras ciencias y con la misma aplicación de las matemáticas. Es muy común que el estudiantado se pregunte constantemente, ¿para qué sirven las matemáticas? Los contenidos de la enseñanza a través de fórmulas, algoritmos y definiciones no bastan para que entiendan el papel que desempeñan las matemáticas en su sociedad, y la situación preocupa cuando no saben para qué se utilizan ni para qué están aprendiendo. Si bien existen metodologías que orientan la práctica docente, no todas permiten el desarrollo de las competencias matemáticas que requiere el alumnado para enfrentar los retos que la sociedad actual demanda. 
Para lograr este fin, Henning y Keune (2007) coinciden con Arrieta et al. (2009) en que se deben proponer situaciones problémicas de la vida social y productiva y que permitan ser representadas a través de los modelos matemáticos, los cuales ayudan al estudiantado a comprender los contenidos de la asignatura, a construir nuevos aprendizajes y a desarrollar competencias matemáticas.

La presente investigación es consecuencia del trabajo docente desarrollado en un proceso de inserción al aula, y su objetivo es motivar una reflexión crítica frente a la práctica de la modelación matemática en Colombia a partir de una experiencia pedagógica. La práctica pedagógica constituyó para el candidato a docente una oportunidad de asumir un rol protagónico y, desde allí, comprender e ir adquiriendo las competencias propias del ejercicio profesional, el cual integra el conocimiento disciplinario y pedagógico, además de las habilidades y cualidades personales y sociales para ejercer la labor docente (Montecinos, Solís, Contreras y Rittershaussen, 2009). Aprovechando ese rol, se busca determinar qué dificultades presenta el alumnado con las tareas de modelación, cómo perciben la utilización de la modelación matemática en el aula, y cuáles acciones conducen satisfactoriamente a la implementación de la modelación matemática en las aulas escolares; dando respuesta a estas interrogantes, se logra el objetivo general del estudio.

\section{REFERENTES CONCEPTUALES}

Se ha discutido en didáctica de las matemáticas sobre las implicaciones de la enseñanza contextualizada, se argumenta que de esta forma se logra un aprendizaje más significativo y el alumnado demuestra mayor interés por la solución de problemas relacionados con su entorno social que con las actividades intramatemáticas. Bassanezi (2002) define la modelación como el arte de convertir problemas de la realidad al lenguaje matemático, resolverlos, interpretarlos y traducir sus soluciones en el lenguaje del mundo real. Por otra parte, Barbosa (2001) entiende la modelación como espacio de indagación y/o investigación en torno a hechos o fenómenos que se originan en otras áreas del mundo real mediada por las matemáticas. En Blomhøj (2004), la práctica que centra el proceso de enseñanza y aprendizaje en la relación entre el mundo tangible y la matemática constituye una práctica de modelación matemática.

Hein y Biembengut (2006) plantean que su ventaja como método de enseñanza e investigación se debe a que permite el diseño de situaciones y actividades para la aprehensión de los conceptos matemáticos en el aula de clase, además de integrar las matemáticas con otras ciencias, la estimulación de la creatividad en la formulación y resolución de problemas y el interés por aplicarlas. Investigadores como García y Rentería (2012), Bassanezi (2002) y Blum y Ferri (2009) argumentan el uso de la modelación y de los modelos en el aula porque ambos cultivan el pensamiento crítico y propositivo del alumnado frente una variedad de acontecimientos de la vida, los hacen ciudadanos y ciudadanas autodidactas y líderes de sus proyectos, como también influyentes en la formación de otras personas de su ambiente escolar y familiar. Aún más, casi todos los fenómenos o problemas del entorno en el cual se desenvuelve el estudiantado cotidianamente son susceptibles de ser descritos y analizados matemáticamente, algo a favor para que se recomiende la implementación de la modelación como recurso en las aulas escolares.

Estas ventajas intrínsecas sirvieron como base para que el Ministerio de Educación Nacional de Colombia [MEN] propusiera la modelación matemática como uno de los cinco procesos generales que deben articular los currículos de matemáticas de la Educación Básica y Media (MEN, 2006). Últimamente se habla de una revolución educativa donde las escuelas tienen autonomía curricular, consistente en mejorar la calidad de la educación atendiendo a las necesidades de las comunidades a las cuales sirven.

No obstante, se han reportado que tales regulaciones no han sido atendidas por el profesorado en sus aulas de clase y que los mecanismos generados para una apropiación e implementación de la modelación matemática en las aulas de clase, parecen ser insuficientes (Villa, 2015, p. 135).

Villa (2015) complementa dicha afirmación apuntando a que "la implementación de la modelación matemática en el cotidiano de las aulas de clase es escasa, debido a que se presentan barreras de diversa 
naturaleza; entre ellas, las altas demandas matemáticas, pedagógicas y personales que la modelación impone al profesorado" (p. 135).

En Colombia, la práctica costumbrista de la mayoría de las instituciones educativas consiste en que el estudiantado de educación básica resuelvan problemas poco contextualizados y gramaticalmente estructurados, a los cuales aplican los algoritmos matemáticos o métodos anteriormente aprendidos, lo que en Estándares Básicos de Competencias en Matemáticas [EBC] colombianos es denominado conocimiento procedimental, para ser exactos, se dedica mucho tiempo a la resolución de problemas.

Por su parte, los Lineamientos Curriculares de Matemáticas en Colombia contemplan cinco procesos generales de las matemáticas: "formular y resolver problemas; modelar procesos y fenómenos de la realidad; comunicar; razonar, y formular, comparar y ejercitar procedimientos y algoritmos" (MEN, 2006, p. 51). En el mismo documento se aclara que la clasificación en cinco procesos generales de la actividad matemática no pretende ser disyunta, es decir, que existen traslapes y relaciones e interacciones múltiples entre ellos; en particular, el modelado matemático es semejante a la resolución de los problemas, pero no idéntico a ello, la modelación comienza con una situación que típicamente no es estructurada. La diferencia principal entre ambos procesos apunta a las competencias en modelación, ni la alumna o alumno más brillante ni la profesora o profesor más competente necesariamente cuenta en su repertorio con los métodos matemáticos oportunos para una situación no estructurada (Blomhøj y Jensen, 2003).

\subsection{Ciclo de la modelación}

La actividad científica del matemático (aplicado) encargado de aplicar y construir modelos explicativos de fenómenos, resolver problemas de las ciencias naturales y sociales o avanzar en una teoría, está reflejada en la modelación en las matemáticas escolares y la fundamenta, es decir, cada docente incorpora en la mentalidad de las y los estudiantes el rigor de las matemáticas en aras de desarrollar en ellas y ellos la intuición y la creatividad y que puedan conocer el mundo en que habitan a través del descubrimiento de la verdad, oculta en sus aplicaciones, motivándoles a ciertas disciplinas o profesiones en un futuro.

En la escuela y el colegio el educador y la educadora deben fomentar la elaboración e interpretación de modelos con el propósito de edificar un concepto matemático dotado de un significado y con la intención de despertar una motivación e interés por las matemáticas debido a la relación que tiene con los problemas del contexto real del alumnado. Freudenthal (1905-1990) precursor de la corriente ideológica conocida como Educación Matemática Realista, pensaba las matemáticas como una actividad humana y sostenía que el núcleo básico del currículo de matemáticas en la escuela debe ser el aprendizaje de estrategias de modelación (o matematización) mediante una serie de acciones o fases de la construcción de modelos (Gravemeijer y Terwel, 2000), como se muestra en la Figura 1. 


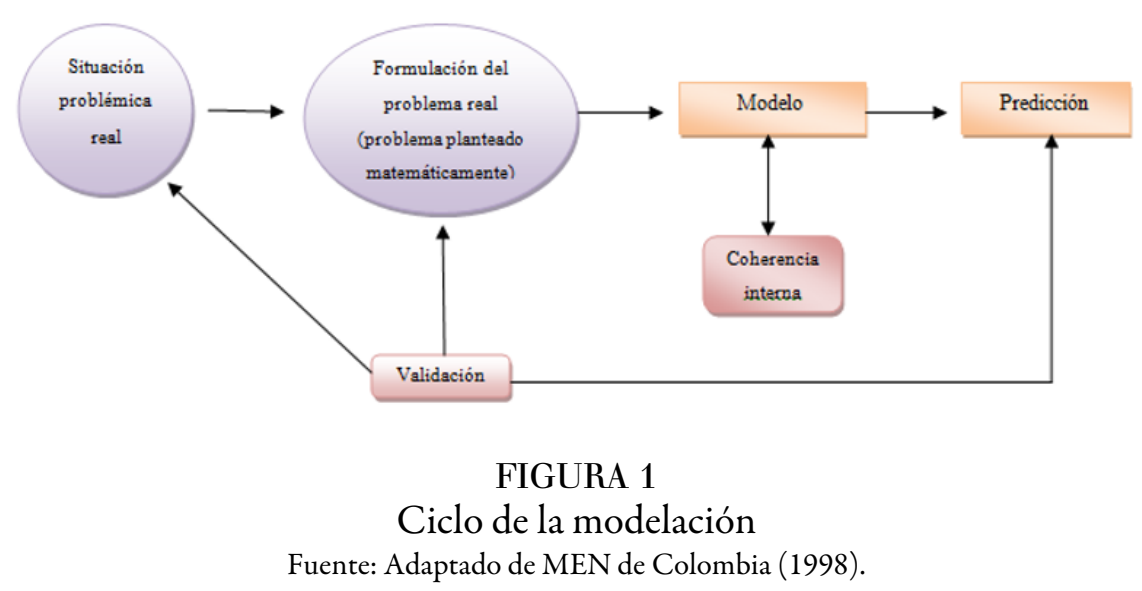

Por consiguiente, la modelación como proceso implica: 1) identificación de la situación, fenómeno o problema de la cotidianidad del estudiantado, 2) establecimiento de los supuestos e hipótesis en que se desarrollará el modelo, 3) formulación matemática del problema, 4) resolución del problema matemático, 5) traducción de las soluciones a la luz del fenómeno, y 6) validación del modelo matemático contrastando los resultados con los datos de la situación contextualizada. Los pasos 2) y 5) dan cuenta del vínculo entre la vida real y las matemáticas.

\section{2. ¿Qué tipos de modelación pueden reconocerse?}

Después de analizar parte de las propuestas reportadas en el campo de la modelación matemática, Villa, Castrillón y Sánchez (2017) reconocen los siguientes tipos de tareas de la modelación matemática:

- Problemas en enunciados verbales: son textos en los que se describe una situación más o menos familiar y se plantea una pregunta cuantitativa; permiten la comprensión de los elementos clave en el enunciado/problema, la construcción de un modelo matemático de los elementos relevantes y las relaciones implicadas en el enunciado, evaluar si el resultado matemático es interpretado de manera adecuada y razonable, y comunicar la solución obtenida del problema original en el mundo real.

- Construcción de representaciones: tareas enfocadas al desarrollo de estrategias de simbolización gráficas y simulación de formas/fenómenos (por ejemplo, utilizando dispositivos electrónicos o software).

- Modelación a través de proyectos: temáticas libres acorde a los intereses del estudiantado donde indaguen/investiguen algunos fenómenos o situaciones de interés, como medio para enseñar un contenido, promover el desarrollo de capacidades, establecer relaciones entre las matemáticas y otras disciplinas, y para el desarrollo de reflexiones y visiones críticas sobre el rol de las matemáticas en la sociedad.

- Uso y análisis de modelos: incorpora diversas acciones, entre ellas el trabajo matemático, la confrontación del modelo con datos extraídos del contexto particular del alumnado, la elaboración de conjeturas sobre posibles extensiones de este, la comprensión de los aspectos que el modelo dice sobre el fenómeno y el análisis de las limitaciones del modelo; este tipo de tareas requiere conocimientos más allá del matemático (interdisciplinario). 


\subsection{Niveles para la implementación de la modelación}

A fin de implementar la modelación en el aula, Barbosa (2001) partiendo de las experiencias relatadas en la literatura especializada, propone tres situaciones, niveles o casos, asociadas con el contexto escolar: la experiencia del personal docente los intereses del grupo estudiantil, entre otros factores, estos se describen en la Tabla 1.

TABLA 1

Formas de implementación de la modelación en el aula.

\begin{tabular}{ll}
\hline Nivel & Descripción \\
\hline I & La/el docente selecciona una situación problémica, la \\
& simplifica si fuera necesario, proporciona los datos \\
& necesarios para resolverla, construye el modelo matemático y \\
& permite que el grupo estudiantil discuta las soluciones del \\
& problema bajo se orientación; en este caso, hay mayor \\
& participación profesoral en la conducción y orientación de las \\
& actividades de modelación. \\
\hline II & Lalel docente propone una situación problémica que debe ser \\
& resuelta por las y los estudiantes con su orientación, \\
& cediéndoles la responsabilidad de simplificar, recolectar los \\
& datos necesarios y proporcionar la solución a la situación \\
& problema; en este caso, existe una menor conducción de las \\
actividades de modelación por parte del profesorado.
\end{tabular}

Fuente: Adaptado de Barbosa (2001).

\section{Procedimientos metodológicos}

La presente investigación tuvo su origen en el trabajo docente desarrollado por el autor de esta en su proceso de inserción al aula, en el contexto de una asignatura de formación profesoral del Programa de Licenciatura de la Universidad del Quindío. Es un estudio de alcance descriptivo el cual utilizó un enfoque de investigación cualitativa que, metodológicamente, centra su atención en descripciones ricas y densas, las que son empleadas para ilustrar, defender o desafiar presupuestos teóricos sostenidos antes de recolectar la información (Hernández, Fernández y Baptista, 2010).

La elección del grupo de trabajo se debió a la recomendación de una colega del autor, la cual facilitó el acercamiento y la autorización de las directivas encabezadas por la rectora del plantel educativo para la aplicación de los instrumentos. Al seleccionar el grupo de trabajo se tuvo en cuenta como criterios el grado de escolaridad y la voluntad de las directivas docentes en colaborar con el estudio. Los sujetos de estudio fueron treinta estudiantes del quinto grado de educación primaria de un colegio en el departamento del Quindío Colombia, cuya población estudiantil es femenina, durante el año lectivo 2017.

En instancia intermedia, hubo concertación con la profesora de matemáticas del grado quinto, y ella concede dos clases de su carga académica para permitir la práctica pedagógica, en las cuales se aplica un instrumento diagnóstico para identificar las dificultades que presentan las estudiantes en la comprensión del concepto de fracción y sus diferentes propiedades, poder diseñar una secuencia de modelación para reforzar en lo requerido, caracterizar el proceso de modelación en este ámbito escolar y evidenciar cómo lo perciben 
las estudiantes. Durante la experiencia se observan los comportamientos de las estudiantes y se elaboran preguntas espontáneas para profundizar en la comprensión de las experiencias pedagógicas en el contexto de inserción al aula. Después de recogida la información (talleres resueltos) al final de cada encuentro, se hace un análisis de contenido para evidenciar aprendizajes y se entrega un reporte a la docente titular.

\section{Resultados}

A partir del análisis efectuado a los instrumentos, se obtuvo que la mayoría de las estudiantes tienen una noción de fracción, pero cuando se les pide que pasen de la representación numérica a la representación gráfica o viceversa, ilustrados en la Figura 2 y la Figura 3, hubo una gran confusión en cuanto a lo que representan el numerador y el denominador de dicha fracción.

(a)

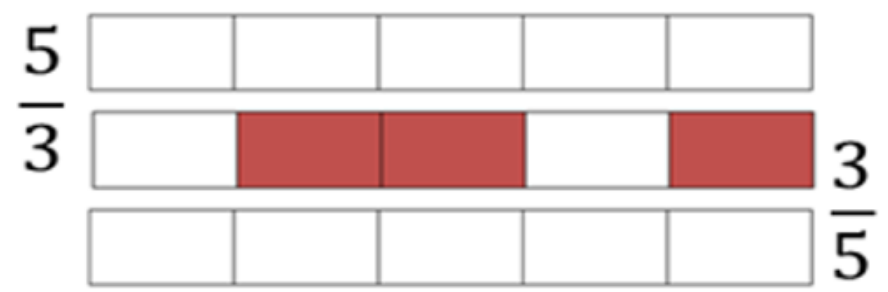

FIGURA 2

Ejercicio pasar de la representación gráfica a la fracción Nota: Las respuestas de las estudiantes se inclinaron mayoritariamente hacia (a) Fuente: Elaboración propia.

(a)

(b)

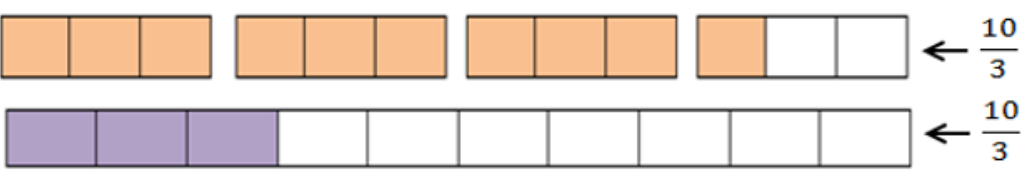

FIGURA 3

Ejercicio pasar de la fracción a la representación gráfica

Nota: Las respuestas de las estudiantes se inclinaron mayoritariamente hacia (b) Fuente: Elaboración propia.

Por ejemplo, Vane dedujo que dicho gráfico representaba la fracción $5 / 3$, y cuando se le pregunta por el procedimiento que ejecutó para su deducción, ella responde: "El procedimiento es que donde había una fracción que eran cinco cuadros y pintaron tres entonces el 5 se pone arriba y el 3 se pone abajo" (comunicación personal, 9 de octubre de 2017), lo cual es equivocado.

La respuesta de Isa fue similar, ella dijo que la fracción representada era $5 / 3$ y en respecto a su procedimiento respondió: "El denominador es lo que uno debe pintar y el numerador es la gráfica” (comunicación personal, 9 de octubre de 2017).

Como ellas, la mayoría de las estudiantes respondieron equivocadamente, y cuando indicaban cual había sido su procedimiento, se pudo identificar que no tenían claro qué representa una fracción, pues en otros ejercicios respondían lo contrario, es decir, en el ejercicio donde debían representar gráficamente la fracción 10/3, Nico hizo el gráfico adecuado y dijo que su procedimiento fue: "Cuando el numerador es más grande la fracción es impropia” (comunicación personal, 9 de octubre de 2017).

En efecto, Nico tiene claro lo que representa una fracción impropia, excepto que en el ejercicio anterior respondió que dicho gráfico representaba la fracción $5 / 3$ y no respondió cual fue el procedimiento a seguir, 
entonces, analizando todo su taller, se concluyó que no tiene completamente claro lo que representa una fracción.

Anie respondió que el gráfico dado representaba la fracción 3/5 y la representación gráfica de la fracción 10/3 fue la adecuada, respecto a su procedimiento dijo: "Yo puse $3 / 5$ porque arriba va la parte pintada y abajo en las partes está dividida la fracción” (comunicación personal, 9 de octubre de 2017).

Pero este desempeño se les atribuye solo a dos de treinta estudiantes, quienes demostraron tener claro lo que representa una fracción. Por lo tanto, en la secuencia que se diseñó, se trabajó el significado de fracción y su representación.

En el diagnóstico elaborado, también se pretendía conocer cuán claro tienen las estudiantes el tema de fracciones equivalentes, planteándose el siguiente problema:

Juan Pablo en su videojuego recorre dos niveles diferentes, en los cuales debe recoger la mayor cantidad de monedas posibles, si en el primer nivel había 50 monedas y Juan pablo recogió 25 monedas, esto es, 25/50 y en el segundo nivel había 10 monedas y juan pablo recogió 5 monedas, esto es, 5/10. ¿En cuál de los dos niveles Juan Pablo tuvo un mejor desempeño? (J. A. Olarte, comunicación personal, 9 de octubre de 2017).

Frente a este interrogante, la respuesta común fue: "El mejor desempeño fue en el primer nivel porque 25>5" (Alu 1, comunicación personal, 9 de octubre de 2017).

Pero también se proporcionó esta respuesta: "Como lo ven es igual, porque hagan las divisiones y ven que es igual, yo las hice y las voy a dejar para que las verifiquen” (Alu2, Comunicación personal, 9 de octubre de 2017).

Hasta aquí se reconoce que las estudiantes no identificaron que las dos fracciones 25/50 y 5/10 eran equivalentes en el problema, por lo que en el siguiente trabajo de campo se comienza por aclarar qué es una fracción, qué representa una fracción y qué son fracciones equivalentes, utilizando las regletas de Cousinaire (Aguilera y Rodríguez, 2017). En la próxima clase se continuó con la segunda actividad, la secuencia de modelación, donde se propuso a las estudiantes participantes una situación problémica cercana a un contexto de consumo, atendiendo a la descripción del segundo nivel descrito en la Tabla 1. La secuencia involucró la situación problema cuyo enunciado se halla en la Figura 4.

1. En un almacén, el costo inicial unitario de una mini Tablet de cierta referencia es de $\$ 200.000$. Si cada mes el costo se incrementa una octava parte del costo inicial, ¿Cuál será el costo en el mes de abril, si la mini Tablet salió a la venta en el mes de enero?

A. Representar el costo de la mini Tablet en cada mes (enero, febrero, marzo, abril) en una tabla de datos.

B. Representar el incremento del costo de la Tablet, en un gráfico.

C. El grafico anterior, nos permite afirmar que el costo de la Tablet:

I. Es creciente de enero a febrero y decreciente de febrero a marzo.

II. Es creciente de enero a abril.

III. Es decreciente de enero a abril.

2. Si las medidas de la mini Tablet que compró Camila son: largo $\frac{61}{3} \mathrm{~cm}$ y ancho $\frac{40}{3} \mathrm{~cm}$, y ella desea guardarla en un protector rectangular de medidas: ancho $21 \mathrm{~cm}$ y largo $15 \mathrm{~cm}$. ¿puede Camila guardarla alli?

\section{FIGURA 4}

Situación problema contemplada en la secuencia de modelación Fuente: Elaboración propia. 


\subsection{Caracterización del proceso de modelación}

A continuación, se analizan ciertos indicadores que caracterizan el proceso de la modelación como vehículo para construir conceptos matemáticos en el aula de clase, de acuerdo a la situación problémica de la Figura 4:

- La estimación suele ser una estrategia suficientemente utilizada en la modelación y la resolución de problemas (Bressan y Bogisic, 1996).

Indicador: Algunas estudiantes escribían 200 en lugar de 200.000, cantidad que multiplicaban por 1/8 para al final de la operación reponerle tres ceros al producto, aparentemente con la intención de desarrollar dicha operación de una manera simple; ver Figura 5.

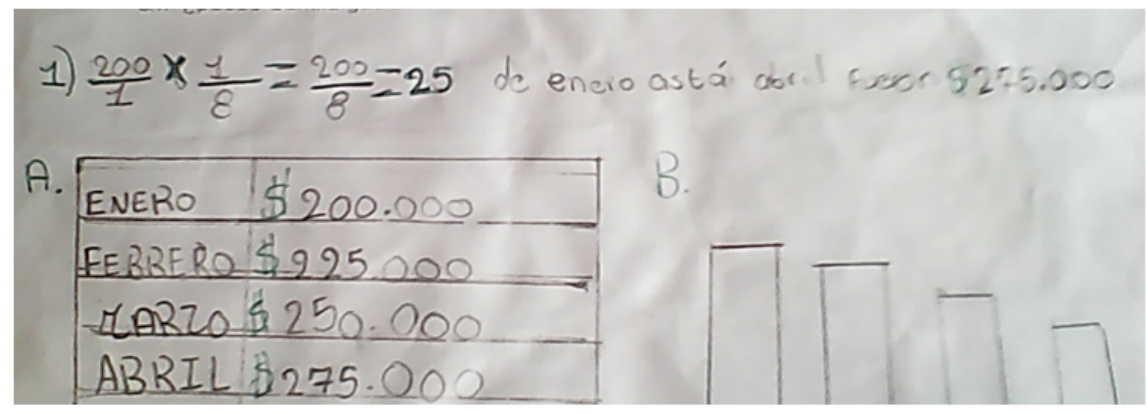

\section{FIGURA 5}

Principio de compensación

Fuente: Elaboración propia.

- En un análisis a posteriori de un fenómeno, el pensamiento divergente orienta la solución del problema modelado (Polya, 2004).

Indicador: Se presentaron casos de estudiantes que usaron el concepto de congruencia y rotación para concluir que la mini Tablet cabe si es girada verticalmente; ver Figura 6.

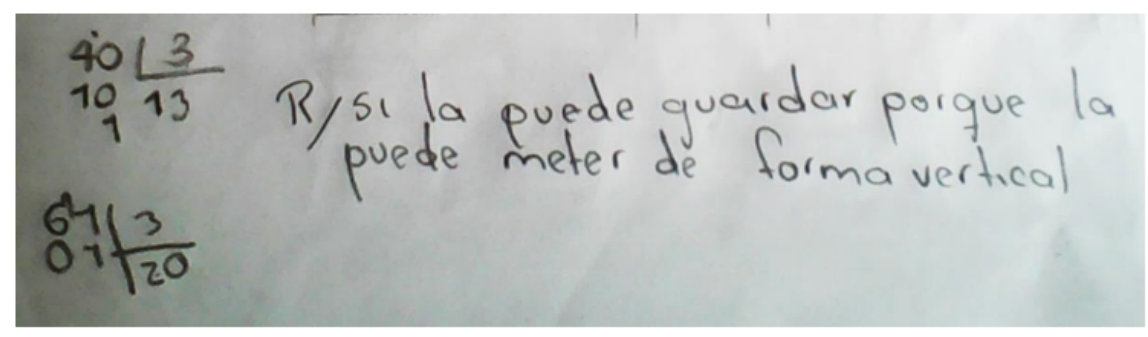

FIGURA 6

Exploración de soluciones

Fuente: Elaboración propia.

- La competencia de modelación implica que el o la estudiante pueda identificar las variables, los parámetros, las relaciones o supuestos en una situación dada del mundo real, para concretarlas en la matemática (Niss y Blum, 2007).

Indicador: Esto se pudo observar en las estrategias que usaron las estudiantes para ejecutar el proceso de modelado, donde implícitamente está el concepto de función, puesto que el precio del producto varía proporcionalmente de un mes al siguiente. Las niñas tomaron el precio inicial del mes de enero, lo dividieron en ocho partes, e hicieron sumas sucesivas del cociente para determinar los incrementos del precio en los meses futuros; ver Figura 7. 


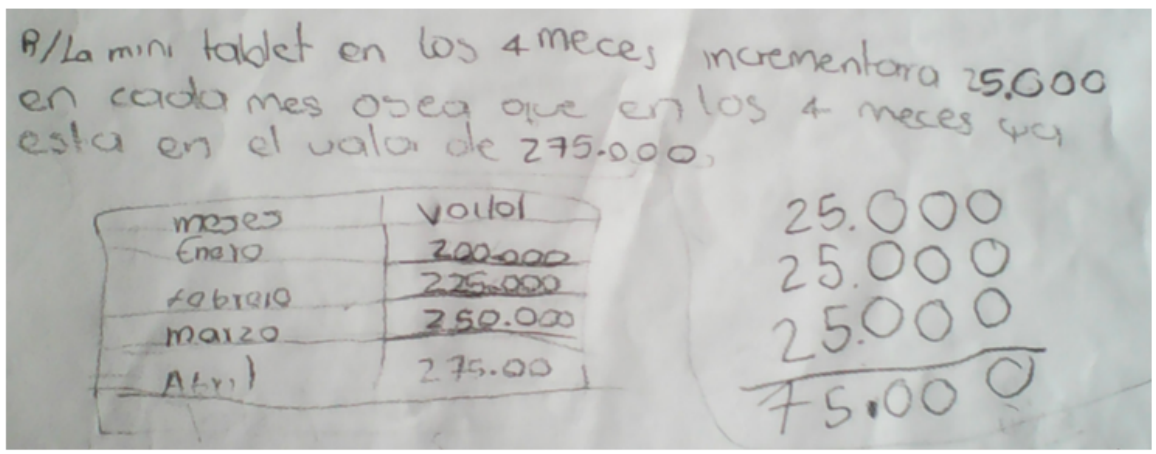

FIGURA 7

Comprensión del problema

Fuente: Elaboración propia.

- La modelación educativa posibilita que las y los estudiantes sean capaces de transitar entre distintos niveles de representación (concreto, pictórico y/o simbólico) (Castellanos y Obando, 2009; Merino, Cañadas y Molina, 2013). Indicador: Combinando los niveles simbólico y pictórico, las y los estudiantes organizan los datos en una tabla de precios de venta y construyen un diagrama de ella con el fin de extraer nueva información acerca del cambio mensual en el precio de la mini Tablet; ver Figura 8.

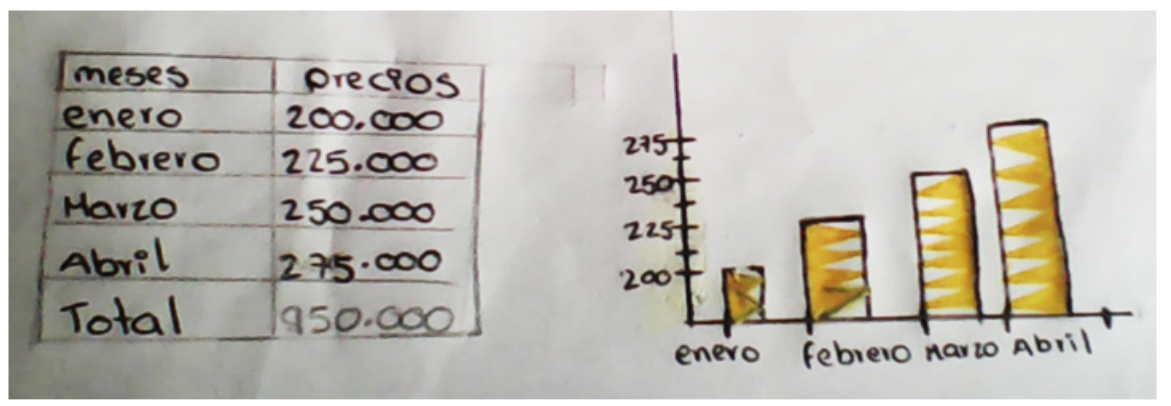

\section{FIGURA 8 \\ Niveles de representación \\ Fuente: Elaboración propia.}

- Un modelo matemático no admite resultados concluyentes contradictorios al mismo problema bajo las mismas hipótesis y restricciones de problema (Abrate, Pochulu y Vargas, 2006).

Indicador: Fue el caso en el cual las estudiantes terminan por responder, con base en el modelo para el numeral 2 y pese a que son modelos equivalentes, que la Mini Tablet puede guardarse en el protector y no puede guardarse en el protector; ver Figura 9. 


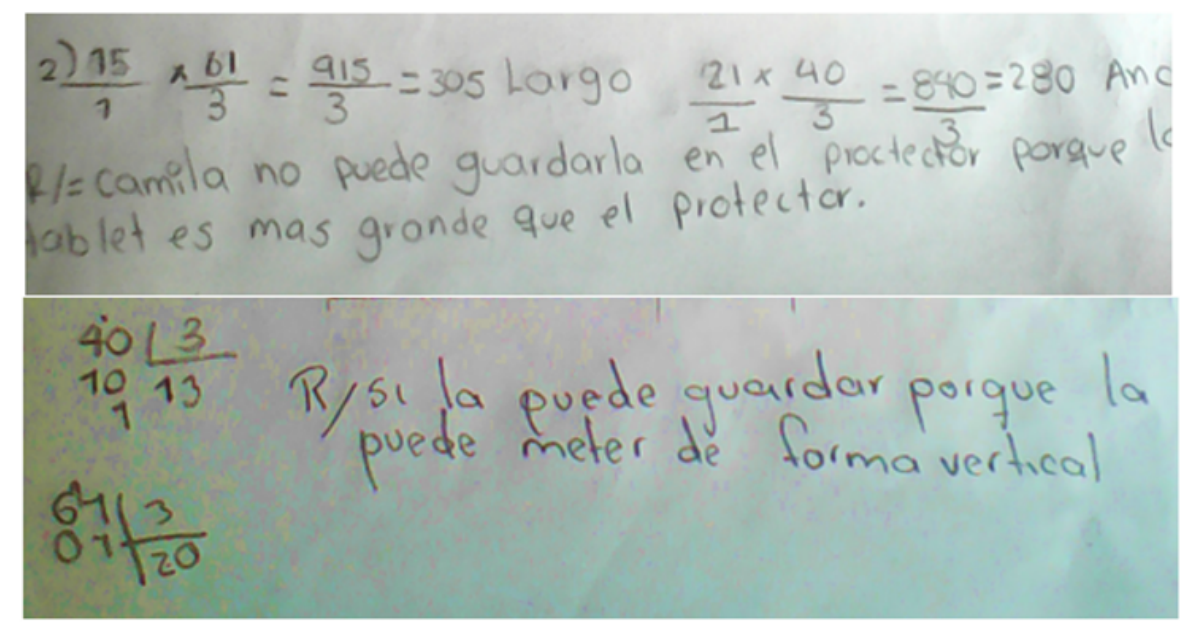

FIGURA 9

Confrontación de soluciones obtenidas por dos estudiantes

Fuente: Elaboración propia.

Con los anteriores indicadores se evidenció que las estudiantes proponen patrones de comportamiento numérico y patrones de comportamiento gráfico, ejecutan cálculos numéricos, organizan la información en tablas, elaboran representaciones gráficas y las interpretan, las cuales son competencias básicas que demanda un buen proceso de modelación, sin embargo, no hubo respuestas muy variadas que significaran nuevos abordajes al problema de la Figura 4, esto debido a que las estudiantes se compartieron sus soluciones en algún momento y unas le copiaron las respuestas a las otras. La Tabla 2 muestra algunos Derechos Básicos de Aprendizaje [DBA] (MEN, 2015) en matemáticas y cómo se evidenciaron estos aprendizajes en las estudiantes. DBA se refieren a un conjunto de saberes y habilidades acerca de lo fundamental que cada estudiante debe aprender al finalizar cada asignatura en cada grado escolar, en concordancia con lo establecido en los EBC y en los lineamientos Curriculares (Gómez, Castro, Bulla, Mora y Pinzón, 2016). 
TABLA 2

Resumen de resultados de los instrumentos aplicados

\begin{tabular}{|c|c|}
\hline$\overline{\mathrm{DBA}}$ & Evidencias de aprendizaje \\
\hline $\begin{array}{l}\text { Interpreta y utiliza los números } \\
\text { naturales y racionales en su } \\
\text { representación fraccionaria. }\end{array}$ & $\begin{array}{l}\text { No interpretan } \\
\text { correctamente la relación } \\
\text { parte - todo cuando la } \\
\text { representan por medio de } \\
\text { fracciones. }\end{array}$ \\
\hline $\begin{array}{l}\text { Compara y ordena números } \\
\text { fraccionarios a través de } \\
\text { diversas interpretaciones, } \\
\text { recursos y representaciones. }\end{array}$ & $\begin{array}{l}\text { Recurren a expresiones } \\
\text { decimales para ordenar } \\
\text { fracciones de mayor a } \\
\text { menor o viceversa. }\end{array}$ \\
\hline $\begin{array}{l}\text { Identifica y describe } \\
\text { propiedades que caracterizan } \\
\text { un cuerpo en términos de la } \\
\text { bidimensionalidad y la } \\
\text { tridimensionalidad. }\end{array}$ & $\begin{array}{l}\text { Reconocen algunas } \\
\text { relaciones inter figurales } \\
\text { para resolver problemas. }\end{array}$ \\
\hline $\begin{array}{l}\text { Describe y desarrolla } \\
\text { estrategias (algoritmos, } \\
\text { propiedades de las operaciones } \\
\text { básicas) para hacer } \\
\text { estimaciones y cálculos. }\end{array}$ & $\begin{array}{l}\text { Utilizan la razón constante } \\
\text { para formar una sucesión } \\
\text { aritmética dado un dato } \\
\text { inicial. }\end{array}$ \\
\hline $\begin{array}{l}\text { Describe e interpreta } \\
\text { variaciones de dependencia } \\
\text { entre cantidades y las } \\
\text { representa por medio de } \\
\text { gráficas. }\end{array}$ & $\begin{array}{l}\text { Organizan la información } \\
\text { en tablas, elaboran } \\
\text { representaciones gráficas y } \\
\text { las interpretan. }\end{array}$ \\
\hline
\end{tabular}

Fuente: Elaboración propia.

\subsection{Percepción de la modelación en el aula}

Hasta el momento la modelación se ha visto como una tarea pasiva. En particular en el estudio de caso, se detectó que las estudiantes mostraban inseguridad, pues dudaban de lo que hacían, y por momentos se estresaban al resolver los ejercicios del primer taller (instrumento diagnóstico). En un diálogo con varias de ellas se interpretó de sus frases que las matemáticas no eran su asignatura favorita, es abstracta y aburrida, porque siempre hay ejercicios difíciles y no saben para qué les sirve eso, que sería bueno experimentarlas en cosas cotidianas de modo que les permita disfrutar de la resolución de los problemas asignados y comprendan los conceptos matemáticos significativamente. ¿Cómo lograr esto? Se debe aterrizar adecuadamente la teoría a las necesidades socioculturales de las y los estudiantes y convertir el aula de clase en un microlaboratorio donde se modelen procesos de la civilización, procurando en la acción evitar la fatiga y el desagrado que produce la memorización de contenidos rígidos y el exceso de ejercicios. Esta percepción de las estudiantes es advertida por Villa, Bustamante, Berrio, Osorio y Ocampo (2008) quienes afirman que:

(...) Sigue predominando una visión de las matemáticas como un área formal y abstracta constituida por definiciones, axiomas e ideas comprimidas y 'exactas' cuya aplicación se encuentra en un conjunto reducido de situaciones artificiales que, en algunos casos, poco o nada tiene que ver con la realidad (p. 41).

Para el segundo taller (modelación) se propuso un problema contextualizado concertado con las estudiantes y se observó mayor interés en las estudiantes por resolverlo, sin embargo, resulta aún más interesante si la elección de los temas cotidianos que se relacionan a los contenidos de aprendizaje incluyen la recolección y sistematización de información que demanda un buen proceso de modelación, y de ser posible, el uso de la tecnología como recurso para la construcción del modelo. 
Los anteriores juicios ponen de relieve que el verdadero papel de la modelación, más allá de lo cognitivo, trata de lo fenoménico, es decir, de lo que se produce en la naturaleza sensible de los y las estudiantes por su interacción con el mundo material (García, 2014).

\section{CONCLUSIONES Y RECOMENDACIONES}

Se pudo constatar fallos conceptuales en las estudiantes principalmente en los temas tratados; cuando se les preguntaba por el significado de fracción o por estrategias distintas para la situación problema propuesta les costaba argumentar; quizás la mayor preocupación que dejó esta experiencia ha sido la popularización de la creencia que las matemáticas son muy difíciles y solo es para superdotados, razón por la cual los encuentros escolares en esta área se tornan aburridos para ellas. La motivación se elevó cuando se habló de problemas prácticos y cotidianos con material alternativo y su disposición cambiaba al imaginarse lo entretenido que sería el trabajo.

En la actualidad, se ha insistido en que, para fomentar actitudes creativas, de resolución de problemas, competencias y pérdida de miedo a la materia se debe utilizar la modelación matemática en el aula (Valdés, 2016). La modelación como metodología de enseñanza requiere de una buena planificación: deben incluirse situaciones problémicas enfocadas en las necesidades del estudiantado, sustentadas en hechos experimentables y no estructurados en totalidad, de forma que el alumnado con el espíritu inherente de investigador consiga la información indispensable para la resolución del problema. No obstante, no se pretende subvalorar la aportación de los algoritmos básicos precursados por el célebre Al-Juarismi y desde entonces impartidos por el profesor de las asignaturas de matemáticas en la enseñanza, en tanto la modelación como proceso sintónico entre la realidad y la teoría, los articula, aunque similar a Masingila, Davidenko y Prus (1996) y Saxe (1988), debe establecerse un equilibrio entre los dos procesos a través de la participación en prácticas culturales matematizables.

En ese sentido, el cuerpo docente en formación tiene en frente la oportunidad de idear estrategias y técnicas paralelas al desarrollo del contenido programático de matemáticas para que cada estudiante aprenda a investigar y elabore modelos matemáticos de sus intereses aplicados en algún área del conocimiento (Mathews y Reed, 2007; Ortiz, 2002). Estas estrategias y técnicas deben influir positivamente en la motivación del estudiantado frente al estudio de las matemáticas y existen autores que recomiendan el uso de herramientas tecnológicas bajo tratamiento pedagógico del profesorado para el estudio de situaciones de contexto que repercutan significativamente en el aula (Arrieta et al., 2009; Murphy, 2003; Palma y Sarmiento, 2015; Salett y Hein, 2004).

La implementación de la modelación matemática en las prácticas educativas sigue siendo bastante heterogénea, debido a que no todo el personal docente en servicio del magisterio colombiano la incluye en el proceso de enseñanza-aprendizaje de las y los estudiantes y hay creación limitada de clubes o semilleros de investigación al interior de las instituciones educativas. Lamentablemente, vista como innovación educativa, la modelación presenta obstáculos individuales y organizacionales para que sea empleada adecuadamente en la enseñanza como: falta de planificación, apoyo insuficiente a la investigación escolar, desarrollo profesional insuficiente del profesorado (capacitación) en este campo (Badilla y Baltodano, 2003).

Finalmente, aquí se ha descrito una experiencia tenida por un profesional en formación, quien expone manifestaciones de sus estudiantes sobre cómo debería enfocarse el proceso formativo y, a partir de ello, reflexiona sobre las competencias necesarias que le permitan generar aprendizajes efectivos en el alumnado, insistiendo en que el reto es: lograr homogeneizar la práctica de la modelación en las instituciones educativas del país, para formar personas más pensantes, más libres y más críticas, algo políticamente ineludible si se pretende alcanzar la Educación de Calidad (MEN, 2011). 


\section{REFERENCIAS}

Abrate, R., Pochulu, M., y Vargas, J. (2006). Errores y dificultades en Matemática: análisis de causas y sugerencias de trabajo. Villa María, Argentina: Universidad Nacional de Villa María.

Aguilera, M. y Rodríguez, S. E. (2017). Uso de las regletas de Cuisenaire para el aprendizaje de las fracciones (Tesis Doctoral). Universidad Pedagógica Nacional, Bogotá, Colombia.

Arrieta, J., Cordero, F., Cârsteanu, A., Mena, J., Rodríguez, R., Romo, A. y Suárez, L. (2009). La modelación y la tecnología en las prácticas de enseñanza de las matemáticas. En Lestón, Patricia (Ed.), Acta Latinoamericana de Matemática Educativa (pp. 1717-1726). México DF, México: Comité Latinoamericano de Matemática Educativa A. C.

Badilla, A., y Baltodano, V. (2003). Algunos factores que limitan el proceso de innovación educativa en la escuela rural: el caso de la escuela Pbro. José Daniel Carmona de Mandayure, Guanacaste. InterSedes: Revista de sedes regionales, 5(8), 1-14. Recuperado de https://www.redalyc.org/articulo.oa?id=66650804

Barbosa, J. C. (2001). Modelagem matemática e os professores: a questão da formação. Bolema, Rio Claro, 14(15), 5-23.

Bassanezi, R. C. (2002). Ensino-aprendizagem com modelagem matemática: uma nova estratégia. São Paulo, Brasil: Editora Contexto.

Blomhøj, M., y Jensen, T. H. (2003). Developing mathematical modelling competence: Conceptual clarification and educational planning. Teaching mathematics and its applications, 22(3), 123-139. doi: https://doi.org/10.1093 /teamat/22.3.123

Blomhøj, M. (2004). Mathematical modelling: a theory for practice. En B. Clarke, D. M. Clarke, G. Emanuelsson, B. Johansson, D. V. Lester, A. Wallby, y K. Wallby (Ed.), International Perspectives on learning and teaching mathematics (pp. 145-159). Göteborg University: National Center for Mathematics Education.

Blum, W., y Ferri, R. B. (2009). Mathematical modelling: Can it be taught and learnt? Journal of mathematical modelling and application, 1(1), 45-58.

Bressan, A., y Bogisic, B. (1996). La estimación, una forma importante de pensar en matemática. Río Negro (provincia), Argentina: Consejo Provincial de Educación.

Castellanos, M., y Obando, J. A. (Octubre de 2009). Errores y dificultades en procesos de representación: el caos de la generalización y el razonamiento algebraico. Conferencia llevada a cabo en el 9o Encuentro Colombiano de Matemática Educativa, Pasto, Colombia.

García, G. I. (2014). Ambiente de aprendizaje: su significado en educación preescolar. Revista de Educación y Desarrollo, (29), 63-72. Recuperado de http://www.cucs.udg.mx/revistas/edu_desarrollo/anteriores/29/029_Garcia.pdf

García, J. J. y Rentería, E. (2012). La medición de la capacidad de resolución de problemas en las ciencias experimentales. Revista Ciência \& Educação, 18(4), 755-767. doi: https://doi.org/10.1590/S1516-731320120 00400002

Gómez, P., Castro, P., Bulla, A., Mora, M. F., y Pinzón, A. (2016). Derechos básicos de aprendizaje en matemáticas: revisión crítica y propuesta de ajuste. Educación y Educadores, 19(3), 315-338. doi: https://dx.doi.org/10.5294 /edu.2016.19.3.1

Gravemeijer, K. P. E., y Terwel, J. (2000). HANS FREUDENTHAL, un matemático en Didáctica y teoría curricular. Journal of Curriculum Studies, 32(6), 777-796. doi: https://doi.org/10.1080/00220270050167170

Hein, N., y Biembengut, M. (Marzo de 2006). Modelaje matemático como método de investigación en clases de matemáticas. En M. Murillo (Presidencia), V festival internacional de matemática. Simposio llevado a cabo en el Colegio Universitario de Puntarenas, Puntarenas, Costa Rica.

Henning H., y Keune M. (2007). Levels of Modelling Competencies. En Blum W., Galbraith P.L., Henn HW., Niss M. (Ed.), Modelling and Applications in Mathematics Education. Boston, MA: Springer.

Hernández, R., Fernández, C., y Baptista, M. (2014). Metodología de la investigación (Vol. 6). México: McGraw-Hill. 
Julián Alejandro Olarte García. Homogeneizar la práctica de la modelación: un reto del Sistema edu...

Masingila, J. O., Davidenko, S., y Prus, E. (1996). Mathematics learning and practice in and out of school: A framework for connecting these experiences. Educational Studies in Mathematics, 31(1-2), 175-200. doi: https://doi.org/ 10.1007/BF00143931

Mathews, S. y Reed, M. (2007). Modelling for pre-service teachers. En C. Haines, P. Galbraith, W. Blum y S. Khan (Ed.), Mathematical modelling (ICTMA 12): Education, Engineering and Economics (pp. 458-464). Chichester, Inglaterra: Horwood Publishing.

Merino, E., Cañadas, M. C., y Molina, M. (2013). Uso de representaciones y patrones por alumnos de quinto de educación primaria en una tarea de generalización. Edma 0-6: Educación Matemática en la Infancia, 2(1), 24-40.

Ministerio de Educación Nacional de Colombia. (2015). Derechos básicos de aprendizaje v2. Bogotá: MEN. Recuperado de http://aprende.colombiaaprende.edu.co/es/node/107746

Ministerio de Educación Nacional de Colombia. (2011). Educación de calidad para la Prosperidad. Colombia: MinEducación. Recuperado de https://www.mineducacion.gov.co/1621/article-89266.html

Ministerio de Educación Nacional de Colombia. (2006). Estándares básicos de competencias en Matemáticas. Bogotá, Colombia: Magisterio. Recuperado de http://www.mineducacion.gov.co/1621/articles-116042_archivo_pdf2 .pdf

Ministerio de Educación Nacional de Colombia. (1998). Lineamientos curriculares de matemáticas. Bogotá, Colombia: Magisterio. Recuperado de http://www.mineducacion.gov.co/1621/articles-339975_matematicas.pdf

Montecinos, C., Solís, M., Contreras, I., y Rittershaussen, S. (2009). Muestras de desempeño docente. Instrumento para evaluar la calidad de la enseñanza y su impacto en el aprendizaje. Santiago: Ediciones Universidad Católica de Chile, UC.

Murphy, C. (2003). Literature review in primary science and ICT. A NESTA Futurelab Research report - report 5.

Niss, M., y Blum, M. (2007). Introducción. En P. L Galbraith, H.W. Henn, M. Niss (Ed.), Modelling and Applications in Mathematics Education (pp. 3-32). New York: Springer. doi: https://doi.org/10.1007/978-0-387-29822-1

Ortiz, J. (2002). Modelización y calculadora gráfica en la enseñanza del Álgebra. Estudio evaluativo de un programa de formación (tesis doctoral). Universidad de Granada, Granada, España. Recuperado de https://dialnet.unirioja. es/servlet/tesis?codigo $=130166$

Palma, C. A., y Sarmiento, R. E. (2015). Estado del arte sobre experiencias de enseñanza de programación a niños y jóvenes para el mejoramiento de las competencias matemáticas en primaria. Revista mexicana de investigación educativa, 20(65), 607-641.

Polya, G. (2004). How to solve it: A new aspect of mathematical method (No. 246). Princeton university press.

Salett, M., y Hein, N. (2004). Modelación matemática y los desafíos para enseñar matemática. Educación matemática, 16(2), 105-125. Recuperado de https://dialnet.unirioja.es/servlet/articulo?codigo $=1159552$

Saxe, G. B. (1988). Candy selling and math learning. Educational researcher, 17(6), 14-21. doi: https://doi.org/10.3 102/0013189X017006014

Valdés, C. E. A. (2016). El desarrollo de la creatividad en la enseñanza de la Matemática. El reto de la educación Matemática en el siglo XXI. Revista Conrado, 12(54), 84-92.

Villa, J. A., Bustamante, C. A., Berrio, M., Osorio, A., y Ocampo, A. (Octubre de 2008). El proceso de modelación matemática en las aulas escolares. A propósito de los 10 años de su inclusión en los lineamientos curriculares colombianos. Curso llevado a cabo en el 9o Encuentro Colombiano de Matemática Educativa, Valledupar, Colombia.

Villa, J. A. (2015). Modelación matemática a partir de problemas de enunciados verbales: un estudio de caso con profesores de matemáticas. Magis. Revista Internacional de Investigación en Educación, 8(16), 133-148. doi: htt ps://doi.org/10.11144/Javeriana.m8-16.mmpe

Villa, J., Castrillón, A., y Sánchez, J. (2017). Tipos de tareas de modelación para la clase de matemáticas. Espaço Plural, $18(36), 219-251$.

CC BY-NC-ND 